\section{THE MICROPHONE}

WE have received the following communications on this subject :-

AT a discussion upon Mr. William Preece's paper on the microphone, which took place before the Society of Telegraph Engineers on Thursday last, the Duke of Argyll called attention to the important part which that invention was likely to play in physiological research. As chairman of the meeting I took occasion to refer to the intimate connection between the microphone and its two elder sisters, the telephone and the "phonograph, in conjunction with which it formed a discovery which would probably be hereafter regarded as one of the greatest achievements in natural science of the present century. I ventured further to draw an anology between the action of the phonograph and the action of the brain in the exercise of memory, and with your permission I will enlarge upon this speculation to the extent of making my reasoning clear enough to submit the same to the critical test.

All impressions received by us from without, either through the tympanum of the ear, the retina of the eye, or through the sensitive nerves of the skin, are, it is generally believed by physiologists, communicated to corpuscular bodies in the brain, which lie embedded in a grey substance, the nature and precise function of which have not yet been fully explained. It would appear that the corpuscular bodies in which the sensitive nerves terminate are connected, through the medium of extremely delicate filaments, with the nervous system of volition, the reaction of the one system upon the other being attributable to mental energy. It may be conceived that any fresh impressions received on the extremely complex sensitive network. of the brain may give rise then and there to acts of volition; but how, it may be asked, can acts of volition arise from impressions that were communicated through the sensitive nerves years before, having been committed in the meantime to what we term the memory? But in order that the mind can deal with an impression previously received it seems necessaryl that it must have the power of reproducing the same from some material record by which the impression has been rendered permanent. Take the case of a tune that we have heard in early youth and which may not have since recurred to us. By some incident or other that tune and the words connected with it become suddenly revivified in the mind. If the tune had been sung into a phonograph it could have been reproduced at any time by releasing a spring moving the barrel of the instrument; and it seems a fair question to ask whether the grey substance of the brain may not, after all, be something analogous to a storehouse of phonographic impressions representing the accumulated treasure of our knowledge and experience, to be called into requisition by the directing power of the mind in turning on, as it were, one barrel or another.

Such a hypothesis might possibly serve also to explain how in sleep, when the directing power of the mind is not active, a local disturbance in the nervous system may turn on one or more phonographic barrels at a time, and thus produce the confused images of dreamland! A powerful mind would exercise a complete control over the innumerable barrels constituting our store of knowledge, whereas in a weak mind the impressions of the past would be brought back into evidence in a confused and irregular manner. Such a supposition might also account for the more vivid recollection of impressions received in early life, when the mechanical record stored up in the brain may be supposed to have been more distinctly and indelibly rendered. In speaking of these impressions as phonographic it does not follow that they were originally conveyed through the tympanum of the ear. Mr.
Willoughby Smith, at the meeting above referred to, called attention to the fact that, by substituting crystalline selenium for carbon in the microphone, a ray of sunlight directed upon the selenium produces a noise comparable with that produced by a Nasmyth hammer; and it is quite feasible that the impressions received through the retina of the eye, and the nerrous system generally, would be equally susceptible of being recorded in the cerebral storehouse. The record itself might be supposed to be of a mechanical, or, more probably, of a molecular character, the one thing important being that it must be material.

These observations are, no doubt, extremely crude, but may serve possibly to direct the attention of physiologists to a point of interest to their science; nor would it be the first occasion on which a phenomenon of inanimate nature had revealed the secrets of animate organisation.

\section{William Stemens}

I HAVE been much interested in your account of the microphone of Prof. Hughes, and I have made, as doubtless many of your readers have also done, the different forms of instrument described by him. The action of the instrument is there apparently attributed to the change of conductivity of the charcoal or carbon or of the mercury globules therein, under the influence of sonorous waves; and whether this is correct is a question worthy of consideration in your columns, and I therefore write more for the purpose of leading others into the inquiry than of making assertions on the subject. My experiments point to another cause, viz., the variation of conducting sectional area of a bad conductor due to the increased or diminished pressure on the point of contact. I am not, of course, referring to the action of the instrument when the vibration is sufficient to absolutely sever the contact, which simply causes the telephone plate to vibrate either in its own period, or some other than that due to the acting sound, as is the case when a musical box is placed on the same table with the instrument; but to the forced or articulate vibrations-the reproduction of the sound acting on the microphone.

Of the several forms of instrument described by Prof. Hughes I have chiefly used that consisting of a rod of charcoal pointed at both ends, supported in a vertical position with its lower point resting in a hollow in a similar piece of charcoal, while its upper end rests against the sides of a similar hollow above. This form is extremely sensitive, and it is difficult to prevent the circuit being broken while having it sufficiently near the source of sound to be reproduced; the sound of a musical box is perfectly rendered, when so far away that there is an absence of jarring from breaks in the circuit; but in talking to the instrument, any rise in the voice breaks contact and produces the jarring sound in the telephone, to the exclusion of all articulation.

I find that any sort of charcoal or carbon will answer, whether soaked with mercury or not; I therefore conclude that the mercury has little or nothing to do with the action. I have tried the effect of sound on rods of carbon and charcoal both saturated with mercury and not so saturated, so arranged that the vibrations could not alter the area of contact, and have obtained no sound whatever from the telephone in the circuit; I therefore conclude that the action takes place at the point or points of contact, and is due to the change of conducting area. To Prof. Hughes is due the credit of inventing a means of varying the electric current with extreme rapidity and slight motion without absolutely breaking the circuit, but I doubt whether a microphone is a proper term for describing the instrument. In gently brushing the stand of the instrument, sound is heard in the telephone, but it does not at all follow that what we hear is a magnified reproduction of the brushing sound; for if the rapidity of the vibrations or motion produced by 